\title{
Fosaprepitant versus aprepitant in the prevention of chemotherapy-induced nausea and vomiting in patients receiving cisplatin-based chemotherapy: a multicenter, randomized, double-blind, double-simulated, positive-controlled phase III trial
}

\author{
Zhonghan Zhang ${ }^{1 \#}$, Yunpeng Yang ${ }^{1 \#}$, Ping $\mathrm{Lu}^{2}$, Xiaoqin $\mathrm{Li}^{3}$, Jianhua Chang ${ }^{4}$, Rongsheng Zheng ${ }^{5}$, \\ Lei Zhou ${ }^{6}$, Shaoshui Chen ${ }^{7}$, Xiaopin Chen ${ }^{8}$, Biyong Ren', Wei Gu ${ }^{10}$, Xiaodong Jiang ${ }^{11}$, Jiyong Peng ${ }^{12}$, \\ Miaolong Huang ${ }^{13}$, Guosheng Feng ${ }^{14}$, Peng Shen ${ }^{15}$, Qingyuan Zhang ${ }^{16}$, Baihong Zhang ${ }^{17}$, Yan Huang ${ }^{1}$, \\ Jingdong $\mathrm{He}^{18}$, Yinglan Chen ${ }^{19}$, Jingxu Cao ${ }^{20}$, Hong Wang ${ }^{21}$, Wei $\mathrm{Li}^{22}$, Huiping Wan ${ }^{23}$, Kejun $\mathrm{Nan}^{24}$, \\ Zijun Liao ${ }^{25}$, Cuiying Zhang ${ }^{26}$, Zhong Lin ${ }^{27}$, Diansheng Zhong ${ }^{28}$, Qing Xu ${ }^{29}$, Hailong Liu ${ }^{30}$, Tao Sun ${ }^{31}$, \\ Yanming Deng ${ }^{32}$, Li Zhang ${ }^{1}$
}

${ }^{1}$ Department of Medical Oncology, Sun Yat-sen University Cancer Center, State Key Laboratory of Oncology in South China, Collaborative Innovation Center for Cancer Medicine, Guangzhou 510060, China; ${ }^{2}$ Oncology Internal Medicine, the First Affiliated Hospital of Xinxiang Medical College, Xinxiang 453000, China; ${ }^{3}$ Oncology Department, Jiangsu University Affiliated Hospital, Zhenjiang 212031 , China; ${ }^{4}$ Medical Oncology, Fudan University Shanghai Cancer Center, Shanghai 200000, China; ${ }^{5}$ Oncology Internal Medicine, the First Affiliated Hospital of Bengbu Medical College, Bengbu 233000, China; ${ }^{6}$ Oncology Department, Beijing Century Temple Hospital, Beijing 100000, China; ${ }^{7}$ Oncology Department, Binzhou Medical University Hospital, Binzhou 256602, China; ${ }^{8}$ Department of Medical Oncology, the First Affiliated Hospital of Chongqing Medical University, Chongqing 400000, China; ${ }^{9}$ Department of Oncology, Chongqing Three Gorges Central Hospital, Chongqing 400000, China; ${ }^{10}$ Department of Respiratory Medicine, Nanjing First Hospital, Nanjing Medical University, Nanjing 210029, China; ${ }^{11}$ Department of Oncology, the First People's Hospital of Lianyungang, Lianyungang 222002, China; ${ }^{12}$ Oncology Department, Jiangxi Pingxiang People's Hospital, Pingxiang 337055, China; ${ }^{13}$ Department of Thoracic Surgery, People's Hospital of Northern Guangdong, Shaoguan 512000, China; ${ }^{14}$ Department of Chemotherapy, the People's Hospital of Guangxi Zhuang Autonomous Region, Nanning 530000, China; ${ }^{15}$ Chemotherapy Ward, the First Affiliated Hospital of Zhejiang University, Hangzhou 310000, China; ${ }^{16}$ First Ward of Internal Medicine of Mammary Gland, Harbin Medical University Cancer Hospital, Harbin 150000, China; ${ }^{17}$ Department of Oncology, the 940th Hospital of Joint Logistics Support Force of People's Liberation Army, Lanzhou 730030, China; ${ }^{18}$ Department of Medical Oncology, Huai'an First People's Hospital, Huai'an 223001, China; ${ }^{19}$ Thoracic Neoplasms, Jiangxi Provincial Cancer Hospital, Nanchang 330000, China; ${ }^{20}$ Oncology Department, Hospital of Armed Police Force, Beijing 100000, China; ${ }^{21}$ Oncology Department, the Third Hospital of Nanchang City, Nanchang 330000, China; ${ }^{22}$ Department of Medical Oncology, the First Bethune Hospital of Jilin University, Changchun 130000, China; ${ }^{23}$ Oncology Department, Jiangxi Provincial People's Hospital, Nanchang 330000, China; ${ }^{24}$ Department of Medical Oncology, Xi'an Jiaotong University the First Hospital, Xi'an 710000, China; ${ }^{25}$ Department of Medical Oncology, Shaanxi Provincial Tumor Hospital, Xi'an 710000, China; ${ }^{26}$ Oncology Internal Medicine, Inner Mongolia People’s Hospital, Huhehaote 010000, China; ${ }^{27}$ Oncology Center, the Fifth Affiliated Hospital Sun Yat-sen University, Zhuhai 519000, China; ${ }^{28}$ The Medical Department, Tianjin Medical University General Hospital, Tianjin 300052, China; ${ }^{29}$ Department of Oncology, Shanghai Tenth People's Hospital of Tongji University, Shanghai 200000, China; ${ }^{30}$ Oncology, Chenzhou No. 1 People's Hospital, Chenzhou 423099, China; ${ }^{31}$ Galactophore Clinic, Liaoning Cancer Hospital and Institute, Shenyang 110000, China; ${ }^{32}$ Department of Cancer Center, Foshan First People's Hospital, Foshan 528000, China

Contributions: (I) Conception and design: L Zhang; (II) Administrative support: None; (III) Provision of study materials or patients: None; (IV) Collection and assembly of data: None; (V) Data analysis and interpretation: Z Zhang, Y Yang; (VI) Manuscript writing: All authors; (VII) Final approval of manuscript: All authors.

"These authors contributed equally to this work.

Correspondence to: Li Zhang, MD. Department of Medical Oncology, Sun Yat-sen University Cancer Center, 651 Dongfeng Road East, Guangzhou 510060, China. Email: zhangli@sysucc.org.cn.

Background: To establish the role of antiemetic therapy with neurokinin-1 (NK-1) receptor antagonists (RAs) in Chinese patients associated with cisplatin-base chemotherapy regimens, this study evaluated the efficacy and safety of single-dose intravenous fosaprepitant-based triple antiemetic regimen to a 3-day 
orally aprepitant-based antiemetic triplet schedule for the prevention of chemotherapy-induced nausea and vomiting (CINV).

Methods: A randomized, double-blind, positive-control design was used to test the noninferiority of fosaprepitant towards aprepitant. Patients receiving cisplatin-base $\left(\geq 50 \mathrm{mg} / \mathrm{m}^{2}\right)$ chemotherapy were administrated palonosetron and dexamethasone with a single-dose fosaprepitant (150 mg on day 1$)$ or a standard aprepitant regimen (125 $\mathrm{mg}$ on day $1,80 \mathrm{mg}$ on day 2 and day 3). The primary endpoint was complete response (CR) during overall phase (OP). Secondary endpoints include CR during acute phase (AP) and delayed phase (DP), no vomiting and no significant nausea during OP, AP and DP. Accrual of 324 patients per treatment arm was planned to confirm noninferiority with expected CR of $75 \%$ and noninferiority margin of minus 10 percentage points.

Results: A total of 648 patients were randomly assigned, and 644 were evaluable for efficacy and safety. Antiemetic efficacy of CR during the OP with fosaprepitant and aprepitant was equivalent $(71.96 \%$ versus $69.35 \%, \mathrm{P}=0.4894)$. And a between-group difference of 2.61 percentage points was finally achieved (95\% CI, -4.42 to 9.64 ) within predefined bounds for noninferiority (primary end point achieved). Both regimens were well tolerated and commonly reported adverse events $(\geq 1 \%)$ were similar between these two group.

Conclusions: Single-dose intravenous fosaprepitant $(150 \mathrm{mg})$ combined with palonosetron and dexamethasone was well tolerated and demonstrated noninferior control of CINV to aprepitant-based triple regimen in Chinese patients treating with cisplatin-base chemotherapy.

Keywords: Chemotherapy-induced nausea and vomiting (CINV); fosaprepitant; aprepitant; neurokinin-1 receptor antagonists; highly emetogenic chemotherapy

Submitted Sep 09, 2019. Accepted for publication Dec 13, 2019.

doi: 10.21037/atm.2019.12.158

View this article at: http://dx.doi.org/10.21037/atm.2019.12.158

\section{Introduction}

Chemotherapy-induced nausea and vomiting (CINV) remains extremely distressing and exerts a negative effect on the quality of patients' lives (1). It has been reported that approximately $60-80 \%$ of cancer patients are inclined to undergo nausea or vomiting caused by chemotherapy, despite effectively preventative antiemetic regimens being used (2). Poorly controlled CINV may lead to unfavorable compliance of chemotherapy and have further adverse impact on survival prognosis (3). For patients receiving highly emetogenic chemotherapy (HEC) and some moderately emetogenic chemotherapy (MEC) regimens, the combination of three drugs, involving 5-hydroxytryptamine type 3 (5-HT3) receptor antagonist (RA), dexamethasone (DXM), and a neurokinin-1 (NK-1) RA, is highly recommended to prevent the CINV $(4,5)$.

Aprepitant was the first substance P/NK-1 RA approved by Food and Drug Administration (FDA) for the treatment of CINV and was commercially available in the market under the brand name $\mathrm{EMEND}^{\circledR}$ as capsules containing
40,80 , or $125 \mathrm{mg}$ of aprepitant for oral administration. The current 3-day schedule for aprepitant-based antiemetic regimen is $125 \mathrm{mg}$ on day 1 followed by $80 \mathrm{mg}$ on days 2 and 3. Nevertheless, the poor water solubility of aprepitant makes it available only in the oral formulation. The defect of aprepitant lie in the fact that a 3-day administration was not convenient enough to cancer patients for good compliance not to mention those certain patients who are unable to receive oral medication. Fosaprepitant is a phosphorylated analog of aprepitant with excellent water-solubility, enabling it rapidly convert to aprepitant after intravenous injection (6). Two phase III studies has demonstrated the superiority of a single dose $150-\mathrm{mg}$ fosaprepitant in enhancing the antiemetic effects of conventional combination of 5-HT3 RA and corticosteroid treatment in comparison with the reciprocal placebo $(7,8)$. Then subsequently, another randomized, double-blind, phase III clinical trial, comparing a single $150-\mathrm{mg}$ dose of intravenous fosaprepitant $\left(\right.$ Emend $^{\circledR}$, Merck and Co. Inc.) with the 3-day regimen of oral aprepitant (Emend ${ }^{\circledR}$, Merck and Co. Inc.), each administered 
Table 1 Study drug schedule

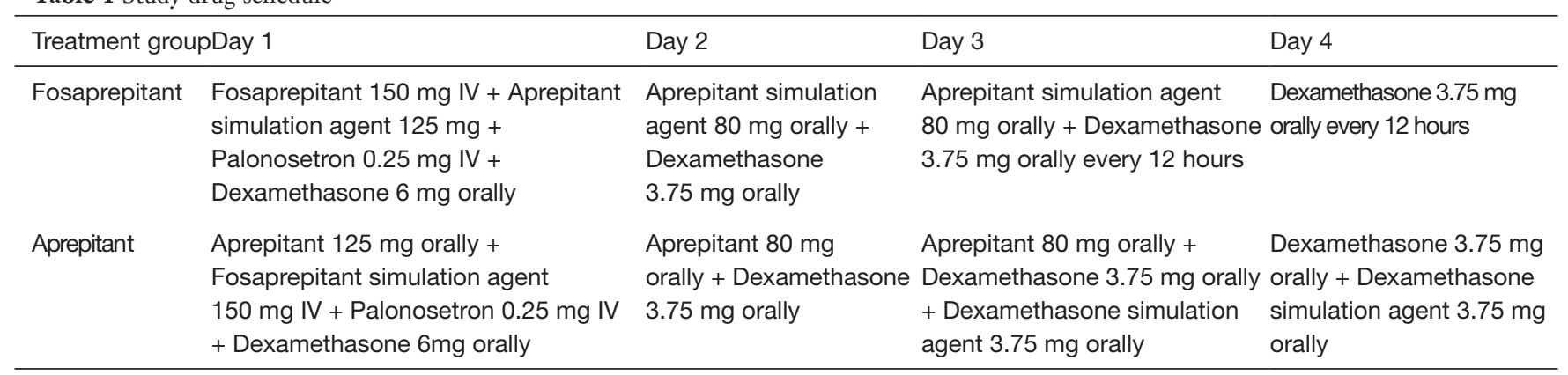

IV, intravenous.

concomitantly with a 5-HT3 RA and a dexamethasone, was performed to demonstrate that the fosaprepitant-based regimen is non-inferior to the aprepitant-based regimen, and the complete response (CR, no vomiting and no use of rescue medication) rates during the overall phase (OP, $0-120 \mathrm{~h}$ after HEC initiation) and delayed phase (DP, $25-120 \mathrm{~h}$ after HEC initiation), were $71.9 \%$ and $74.3 \%$ in fosaprepitant treatment group and $72.3 \%$ and $74.2 \%$ in patients treated with aprepitant respectively, which showed no statistical significance between these two regimens (9). Similar efficacy and safety results were reported in Indian and Japanese population $(10,11)$. And therefore, for its equivalent efficacy and comparable safety profile, fosaprepitant-based triplet was recommended as one of the standard antiemetic regimen in NCCN and ESMO antiemetic guideline since $2008(4,5)$.

At present, the prices of fosaprepitant in the United States is about $\$ 342.81-\$ 398.36$ for a supply of one powder for injection $150 \mathrm{mg}$ (https://www.rxusa.com). Since it is not at a low price for the majority of cancer patients, the increasing cost of escalating antiemetic prophylaxis as supporting therapy needed to be taken into consideration. With the emergence of various novel agents (such as antiemetics) for supportive treatment, a rising burden has been placed on both of the patient individual and the whole health system. Recent years, value-based cancer care has been highlighted in oncology and drawing more and more attention to better assessing the value of various cancer treatments (12-14). Research and development into highquality generic drug is an important means to ensure the efficacy as the reference drug while providing affordable price, especially in developing country.

Nowadays, there is no commercially available fosaprepitant for drug market in China to fulfill the urgent clinical demand. A study investigating the fosaprepitant
(Chia Tai Tianqing Pharmaceutical Group Co., Ltd) versus aprepitant in Chinese patients demonstrating that CR in the fosaprepitant group was not inferior to that in the aprepitant group (90.85\% versus $94.17 \%, \mathrm{P}=0.1302$ ) during OP, however, the CR rates of this study seemed higher than the historically reported results with these outcomes. Since there has limited data evaluating the efficacy and safety profile of generic fosaprepitant in Chinese patients population so far, therefore we conduct this present multicenter, randomized, double-blind, doublesimulated, positive-control, phase III study in China to further conform the antiemetic efficacy and safety for of fosaprepitant (Jiangsu Hansoh Pharmaceutical Group Co., Ltd.) plus palonosetron and dexamethasone in patients receiving HEC in CINV control.

\section{Methods}

\section{Study design}

This multicenter, randomized, double-blind, doublesimulated, positive-control, phase III study trial was performed in conformance with Good Clinical Practice guidelines in China between October 2014 and November 2015 after approval of each site's institutional review board (approved ID: 2014-xy-060). The study protocol was approved by the Ethics Committee Review Board of Sun Yat-sen University (approved ID: A2014-058) as well as other 38 participating center and was registered with www. chinadrugtrials.org.cn (approved ID: CTR20150598).

Patients firstly stratified by gender and then based on whether the first administration of chemotherapy or not and the emetogenic potential of anticancer agents (excluding cisplatin) were randomized to different treatment groups in accordance with the computer-generated, blinded allocation schedule (Table 1). Both patients and researchers 
were blinded to the therapeutic groupings. Moreover, with a double-simulated design, each treatment group received either intravenous fosaprepitant in combination with matched oral aprepitant simulation agent or oral aprepitant plus with matched intravenous fosaprepitant simulation agent to ensure absolutely double blinding. Patients were centrally randomized, using clinical research central randomization system managed by Nanjing Medical University, and allocated in 1:1 ratio to experiment arm and control arm. The balance of stratification factors including history of chemotherapy (ever, never), gender (male, female) and emetogenic potential of chemotherapy agents other than cisplatin (highly emetogenic, non-highly emetogenic) will be ensured. Random assignment form was generated by PLAN process using the Statistical Analysis System (SAS) software by statistician from Nanjing Medical University. The patient was required to receive the allocated drug treatment within 48 hours after randomization.

The placebo agent has an identical appearance to the experimental drug. Aprepitant were provided by Merck and Co. Inc. Study drugs are coded and loaded into the central randomization system with random allocation schedule as blind codes. Personnel in the participating center, patients, sponsor and staff involved in the clinical trial are masked to the treatment regimen of each patient. Patients recorded episodes of vomiting, related rescue therapy, and daily nausea evaluations from the beginning of administration of chemotherapy (0 hours) until the morning of day 6 (120 hours). Enrollment, randomization, and intervention assignment was carried out by two personnel involved with the study at the Department of Medical Oncology of Sun Yat-sen University.

The dosage and schedule of palonosetron and dexamethasone (DXM) were administered according to the current prescribing information. Based on a former study conducting in Chinese population, the DXM administration of $6 \mathrm{mg}$ on day $1,3.75 \mathrm{mg}$ on the following day 2 to 4 was considered acceptable and sufficient (15). In this study, the dosage of DXM was $6 \mathrm{mg}$ on day 1 followed by $3.75 \mathrm{mg}$ on day 2 and $3.75 \mathrm{mg}$ orally every 12 hours on day 3 to 4 in fosaprepitant group. And in aprepitant group, the dosage of DXM was $6 \mathrm{mg}$ on day 1 followed $3.75 \mathrm{mg}$ on day 2 to 4, with a DXM simulation agent $3.75 \mathrm{mg}$ orally on day 3 to 4 . Due to DXM systemic exposure being increased by aprepitant (16), doses of DXM in participants administered aprepitant regimen group was reduced on day 3 to 4 . And this regimen of DXM was in accordance to the former study on evaluation of aprepitant in Chinese population (15).
Complete response (CR), defined as no vomiting and no use of rescue therapy, in the overall phase (OP) was the primary efficacy endpoint of this study. Noninferiority of the fosaprepitant group compared with the aprepitant group was determined if the lower limit of the $95 \%$ confidence interval (CI) was 10 percentage points for the difference in reaction rates of the two regimens. The secondary efficacy endpoints included the proportions of patients who either achieved complete response in the acute phase (AP, 0-24 h after HEC initiation) and DP, no vomiting and significant nausea during the OP, AP and DP. Both primary and secondary hypothesis tests were done according to the comparison of the lower limit of the $95 \%$ CI for the difference between fosaprepitant and aprepitant groups to predefined noninferiority margins.

\section{Patients}

Male and female patients aged $\geq 18$ and $\leq 75$ years old with histologically confirmed solid malignant tumors, Eastern Cooperative Oncology Group (ECOG) Performance Status of $0-2$, and predicted life expectancy of $\geq 3$ months, who were scheduled for a single day of cisplatin (dosage $\geq 50 \mathrm{mg} / \mathrm{m}^{2}$ and infusion time $\leq 3$ hours) were eligible. The major exclusion criteria were as follows: (I) patients were mentally disable or suffered from emotional disorders; (II) patients were current illicit drug use, including alcohol abuse; (III) patients scheduled administration of stem cell rescue therapy during cisplatin chemotherapy; (IV) patients have participated in other clinical trials in the past 4 weeks; (V) patients were treated with chemotherapy including ordinary paclitaxel (using cator oil as a solvent); (VI) patients had an active infection or uncontrolled disease other than malignancy; (VII) patients were scheduled for multiday cisplatin chemotherapy; (VIII) patients were treated with moderate or highly emetogenic chemotherapy within 6 days prior to the initial of cisplatin infusion and/or 6 days after cisplatin infusion; (IX) patients were scheduled to receive radiation therapy to the abdomen or pelvis within a week of treatment; (X) Absolute neutrophil count $<1,500$ cells/ L, WBC count $<3,000$ cells/ L, platelet count $<100,000$ cells/ L, AST and ALT $>2.5$ upper limit of normal (ULN), bilirubin $>1.5 \mathrm{ULN}$, and creatinine $>1.5 \mathrm{ULN}$; (XI) Patients were pregnant or breastfeeding; (XII) patients taking systemic corticosteroids not including topical and inhaled corticosteroids; (XIII) patients had suffered from vomiting or nausea in the 24 hours before treatment. Before the study started, all participants provided written informed 
consent after being explained the study procedures and potential risks. In addition, each subject was assured of his/ her freedom to terminate study participation at any time.

\section{Study visits and evaluation}

Assessments of efficacy, tolerability and safety variables were performed within 5 days after the initial of chemotherapy $(0-120 \mathrm{hr})$, including the acute and delayed phase. Patients self-recorded the vomiting and retching episodes, daily nausea evaluations based on a $100-\mathrm{mm}$ visual analog scale (VAS) (17), and the use of rescue therapy for 5 days after the initial of chemotherapy. Notably, Functional Living Index-Emesis (FLIE) is an effective measurement standard for impact of CINV on daily life, including nine questions about nausea and nine about vomiting (18), what's more, "no impact of CINV on daily life" was defined as mean scores $>6$ on a scale of 7 (>108 in total).

The safety evaluations were vital signs, adverse events (AEs), severe adverse events (SAEs), electrocardiograms (ECG) and general laboratory tests. The study point (vomiting and nausea) would not be considered as adverse events unless they resulted in hospitalization or were defined as SAEs by the researchers. Clinical AEs were measured on the basis of National Cancer Institute Common Toxicity Criteria, version 4.0.

\section{Statistical analysis}

Assuming a two-sided 5\% significance level for testing the primary efficacy hypothesis and an expected CR rate of $75 \%$ with a difference between treatment groups of $\leq 10 \%$, 294 evaluable patients per regimen would yield $80 \%$ power to determine noninferiority for by using a noninferiority margin of 10 percentage points. Assuming a $5 \%$ dropout rate, 648 patients needed to be enrolled.

Statistical analyses were performed in the safety set (SS; all patients who received at least one dose of study treatment), the full analysis set (FAS; all SS patients who had $\geq 1$ efficacy assessment) and the per protocol set (PPS; all FAS patients who had no protocol violations that directly affected the primary endpoint). The primary and secondary efficacy endpoints were evaluated with FAS and PPS.

The baseline demographic characteristics of two groups were analyzed, among which the measurement data were tested by $t$-test, the grade data by Wilcoxon test, and the classification data by $\chi^{2}$ test or Fisher's exact probability method. For primary endpoint, non-inferiority test was performed on the proportion of patients with CR in OP $(0-120 \mathrm{~h})$ after chemotherapy in the two regimen groups. The non-inferiority threshold value was $-10 \%$. If the lower bound of $95 \%$ CI of complete response within 5 days is greater than $-10 \%$, it will be considered non-inferior. For secondary endpoints, Fisher's exact probability method was used for $\mathrm{CR}$ in $\mathrm{AP}$ and $\mathrm{DP}$, no vomiting and no significant nausea during OP, AP and DP. Fisher's exact or Chi-square test was used to compare variables between treatment groups for different AEs by CIs. Kaplan-Meier curves of time to first vomiting were constructed for both groups. A two-sided $\mathrm{P}<0.05$ was considered statistically significant.

\section{Results}

\section{Patients}

Of 648 patients screened for inclusion, 4 were excluded for no study drug being used (Figure 1). The remaining 644 patients were stratified by gender, the first administration of chemotherapy and the emetogenic potential of anticancer agents (excluding cisplatin) were randomized into two treatment groups. Both two groups had similar baseline demographics (Table 2). There were no significant differences in risk factors involving age, gender, ECOG PS score, proportion of initial chemotherapy, alcohol use, allergy history, motion sickness history, cancer treatment history, vomiting related to pregnancy, and other observational indicators between the two groups (all $\mathrm{P}>0.05)$.

\section{Efficacy}

For primary endpoints, CR in the OP of FAS were displayed with $71.96 \%$ (95\% CI, $67.90 \%$ to $76.87 \%$ ) in the fosaprepitant regimen group and 69.35\% (95\% CI, 64.32\% to $74.38 \%$ ) in the aprepitant regimen group, respectively $(\mathrm{P}=0.4894$; Figure 2$)$, and a between-group difference of 2.61 percentage points was finally achieved (95\% CI, -4.42 to 9.64; Figure 3). The lower limit of $95 \%$ CI for the betweengroup difference is $-4.42 \%>-10 \%$ (non-inferior limit). In PPS, the lower limit of $95 \%$ CI of the between-group difference was $-5.60 \%(>-10 \%)$, which was consistent with FAS.

For secondary endpoints, FAS outcome of CR in the AP were $90.97 \%$ (95\% CI, $87.83 \%$ to $94.11 \%$ ) in the fosaprepitant group while $86.69 \%$ (95\% CI, $82.99 \%$ to $90.39 \%)$ in the aprepitant treatment group $(\mathrm{P}=0.1036$; 


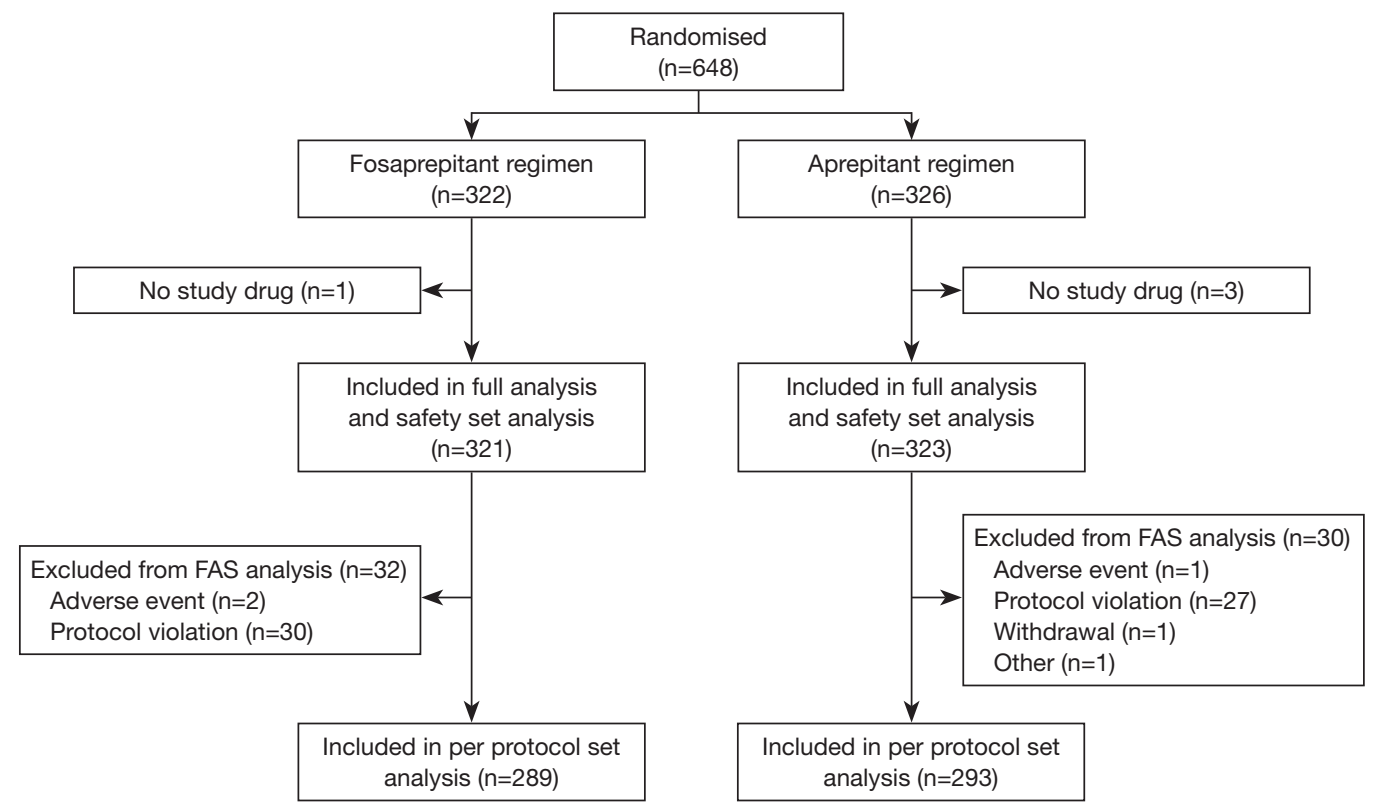

Figure 1 CONSORT diagram. The efficacy and safety analysis populations included patients who received at least one dose of study therapy, received cisplatin-based chemotherapy, and had at least one post-treatment efficacy assessment. FAS, full analysis set; SS, safety set; PPS, per protocol set.

Table 2 Baseline demographics and specific clinical characteristics (FAS population)

\begin{tabular}{|c|c|c|c|}
\hline Characteristic & Fosaprepitant regimen $(n=321)$ & Aprepitant regimen $(n=323)$ & $P$ value \\
\hline Male & $191(59.50)$ & $191(59.13)$ & \multirow[t]{2}{*}{0.9362} \\
\hline Female & $130(40.50)$ & $132(40.87)$ & \\
\hline \multicolumn{4}{|l|}{ Age, years } \\
\hline Range & $18-75$ & $25-75$ & $0.9993^{\mathrm{a}}$ \\
\hline \multicolumn{4}{|l|}{ ECOG PS, n (\%) } \\
\hline 0 & $104(32.40)$ & $111(34.37)$ & \multirow[t]{2}{*}{$0.3487^{b}$} \\
\hline 1 & $204(63.55)$ & 207 (64.09) & \\
\hline History of cancer treatment, $\mathrm{n}(\%)$ & $175(54.52)$ & $176(54.49)$ & 1.0000 \\
\hline History of alcohol use, $\mathrm{n}(\%)$ & $88(27.41)$ & $76(23.53)$ & 0.2782 \\
\hline History of motion sickness, $\mathrm{n}(\%)$ & 19 (5.92) & $9(2.79)$ & 0.0553 \\
\hline History of vomiting during pregnancy ${ }^{\star}, \mathrm{n}(\%)$ & $47(14.64)$ & $52(16.15)$ & 0.7544 \\
\hline History of vomiting during anti-tumor treatment, $\mathrm{n}(\%)$ & $59(18.38)$ & $71(21.98)$ & 0.2805 \\
\hline History of drug allergy, $\mathrm{n}(\%)$ & $23(7.17)$ & $24(7.43)$ & 1.0000 \\
\hline
\end{tabular}



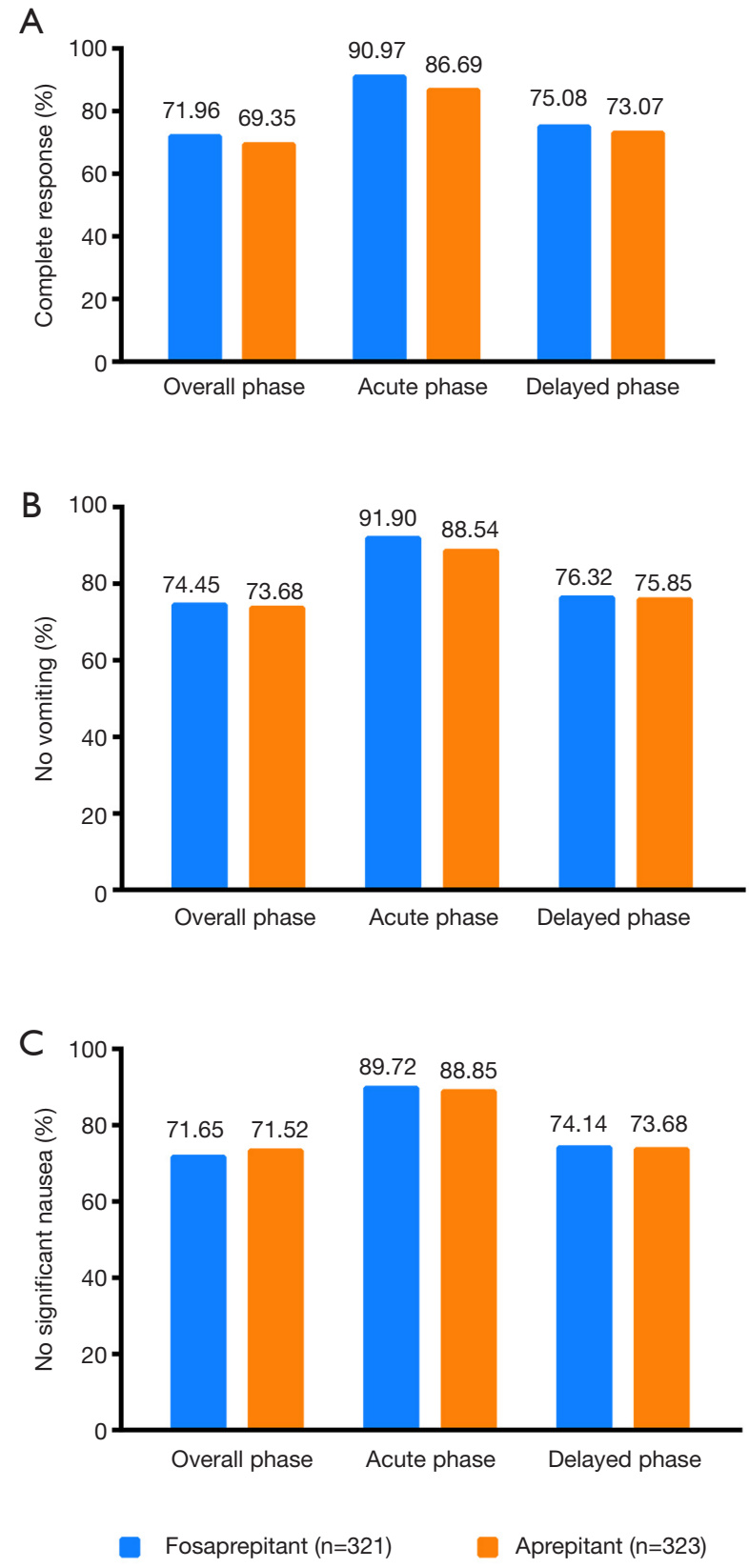

Figure 2 Complete response (CR), no vomiting (NV) and no significant nausea by phase. Bar graph shows percentage of patients achieving (A) CR and (B) NV and (C) no significant nausea end points during the 120 hours after initiation of chemotherapy. CR was defined as NV and no use of rescue medication. Blue bars represent single-dose fosaprepitant regimen; orange bars, 3-day aprepitant regimen in this study. Overall phase was 0 to 120 hours after initiation of chemotherapy. Acute phase was 0 to 24 hours after initiation of chemotherapy. Delayed phase was 25 to 120 hours after initiation of chemotherapy.

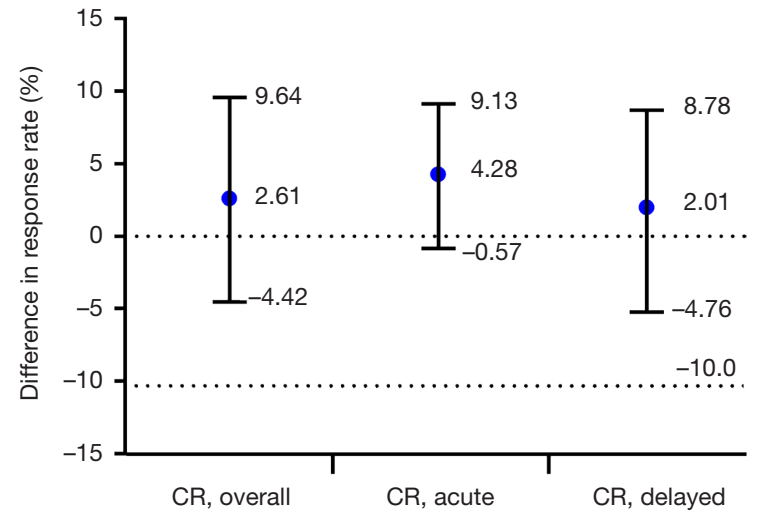

Figure 3 Differences between complete response (CR) end points (between fosaprepitant regimen and aprepitant regimen) during the 120 hours after chemotherapy administration (error bars, $95 \% \mathrm{CI})$. CR is defined as no vomiting and no use of rescue medication. Overall phase was 0 to 120 hours after initiation of chemotherapy. Delayed phase was 25 to 120 hours after initiation of chemotherapy. 95\% CIs were around the difference in response rates calculated by the Miettinen and Nurminen method. Dashed line represents prespecified lower bound of 95\% CI for noninferiority.

Figure 2A). In addition, for CR in the DP, $75.08 \%$ (95\% CI, $70.35 \%$ to $79.81 \%$ ) in the fosaprepitant group compared with $73.07 \%$ (95\% CI, $68.23 \%$ to $77.91 \%$ ) in the aprepitant treatment group were observed $(\mathrm{P}=0.5900$; Figure $2 A)$. For FAS outcome of no vomiting, in the OP, the rates of were $74.45 \%$ (95\% CI, $69.68 \%$ to $79.22 \%$ ) of patients in the fosaprepitant group and $73.68 \%$ (95\% CI, $68.88 \%$ to $78.48 \%)$ in the aprepitant group $(\mathrm{P}=0.8575$; Figure $2 B)$. During the AP and DP, the cases of no vomiting were reported similar in these two groups $(91.90 \%$ vs. $88.54 \%$, $\mathrm{P}=0.1845$ for the AP and $76.32 \%$ vs. $75.85 \%, \mathrm{P}=0.9265$ for the DP; Figure 2B). For FAS outcome of no significant nausea (VAS <25), in the OP, the rates were $71.65 \%$ (95\% CI, $66.72 \%$ to $76.58 \%$ ) of patients in the fosaprepitant group and $71.52 \%$ (95\% CI, $66.60 \%$ to $76.44 \%$ ) in the aprepitant group $(\mathrm{P}=1.000$; Figure $2 C)$. During the $\mathrm{AP}$ and $\mathrm{DP}$, the cases of no significant nausea were also reported similar in these two groups $(89.72 \%$ vs. $88.85 \%, \mathrm{P}=0.7991$ for the $\mathrm{AP}$ and $74.14 \%$ vs. $73.68 \%, \mathrm{P}=0.9286$ for the $\mathrm{DP}$; Figure 2C). Kaplan-Meier curves of time to first vomiting failure were similar between the two groups $(\mathrm{P}=0.8110$, Figure S1). 


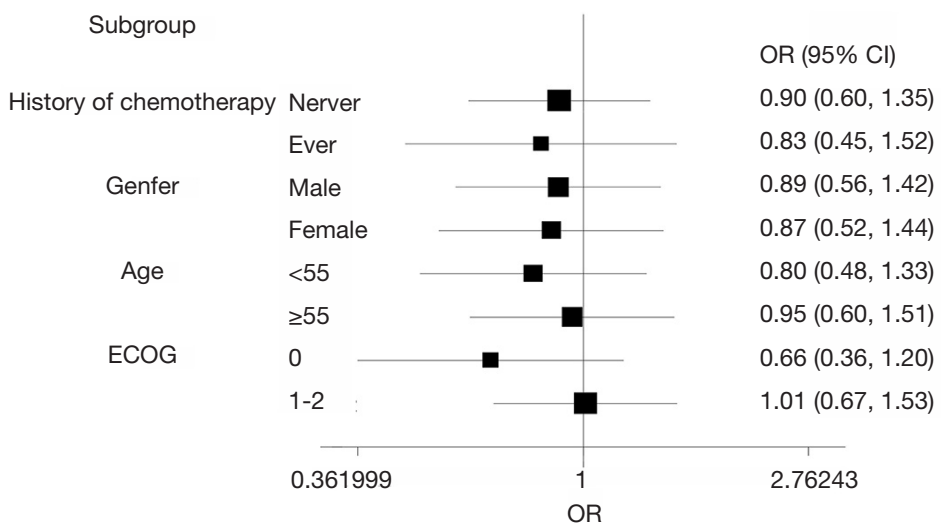

Figure 4 Subgroup analysis of complete response (CR) in overall phase (OP). OR, odds ratio; CI, confidence interval; ECOG, Eastern Cooperative Oncology Group.

The change of FLIE score before and after treatment in the fosaprepitant group $(-9.73 \pm 21.55)$ and aprepitant group $(-9.93 \pm 18.76)$ were statistically significant $(\mathrm{P}<0.0001)$. And there was no significant difference in the change of FLIE score before and after treatment between these two groups $(\mathrm{P}=0.9008)$. According to the results of FAS set analysis, the degree of nausea (VAS score) of patients in the fosaprepitant group was $7.06 \pm 17.30,9.13 \pm 18.33,10.39 \pm 19.23,9.06 \pm 18.76$ and $7.77 \pm 17.50$ on the first to the fifth day after treatment, respectively, while those in the aprepitant group were $6.86 \pm 15.86,9.53 \pm 18.95,10.54 \pm 19.60,8.25 \pm 16.97$ and $7.34 \pm 16.42$, respectively. There was no significant difference between these two groups $(\mathrm{P}>0.05)$. And the PPS analysis were all consistent with FAS in secondary endpoints (data not shown).

The predefined subgroup analyses included history of chemotherapy (never, ever), gender(male, female), age ( $<55$, $\geq 55)$, ECOG $(0,1-2)$. There was no significant difference in terms of $\mathrm{CR}$ rate in $\mathrm{OP}(\mathrm{P}>0.05)$ (Figure 4). Detailed results were listed in the Supplementary File (Table S1).

\section{Tolerability}

Finally, 644 patients (321 in fosaprepitant group and 323 in aprepitant group) were included in safety set (SS) analysis with 4 patients absent of study drug. The incidence of AEs was $85.05 \%(273 / 321)$ in the fosaprepitant group and $83.90 \%(271 / 323)$ in the aprepitant group. Adverse reactions were $25.86 \%(83 / 321)$ and $27.24 \%(88 / 323)$, respectively. SAEs were $1.87 \%(6 / 321)$ and $2.48 \%(8 / 323)$ respectively. SAEs occurred in 6 cases in fosaprepitant group while 9 cases in aprepitant group, which was considered all irrelevant to the study drugs.

The commonly reported AEs $(\geq 1 \%)$ in fosaprepitant group were hiccup $(27 / 321,8.41 \%)$, constipation (23/321,7.17\%), decreased appetite/anorexia (8/321, 2.49\%), fatigue $(7 / 321,2.18 \%)$, dizziness $(7 / 321,2.18 \%)$, abdominal distension (6/321, 1.87\%) and vertigo (4/321, 1.25\%). The commonly reported AEs $(\geq 1 \%)$ in the aprepitant group were hiccup $(23 / 323,7.12 \%)$, constipation $(26 / 323,8.05 \%)$, decreased appetite/anorexia (9/323, 2.79\%), fatigue (9/323, $2.79 \%)$, dizziness $(9 / 323,2.79 \%)$, abdominal distension (9/323, 2.79\%), and diarrhea (4/323, 1.24\%). The AEs profile for the fosaprepitant $150-\mathrm{mg}$ treatment was in accordance with that for the 3-day aprepitant regimen, there were no significant differences in the overall occurrence of AEs between two treatment arms (Table 3).

\section{Discussion}

Despite remarkable advancements in control of CINV, there is urgent need for more effective approaches and better compliance of comprehensible guidelines $(19,20)$. Previous studies have established that 3-day oral aprepitant have potent efficacy in preventing CINV during the acute and delayed phases when combined with 5-HT3 RA and dexamethasone $(21,22)$. Comparing to the present 3 -day aprepitant regimen, single-dose intravenous fosaprepitant provides more convenience without wakening the antiemetic effects. This current study was aimed to evaluate the antiemetic efficacy and safety profile of the fosaprepitant-based regimen in Chinese patients. Notably, this was a randomized, double-blind and double-simulated positive-control phase III clinical trial of the single-dose 
Table 3 Summary of adverse events

\begin{tabular}{|c|c|c|c|}
\hline & Fosaprepitant regimen $(n=321)$ & Aprepitant regimen $(n=323)$ & $P$ value \\
\hline Adverse reactions & $83(25.86)$ & $88(27.24)$ & 0.7215 \\
\hline Serious adverse events & $6(1.87)$ & $8(2.48)$ & 0.7882 \\
\hline \multicolumn{4}{|c|}{ Commonly reported AEs ( $\geq 1 \%$ of subjects) } \\
\hline Constipation & $23(7.17)$ & $26(8.05)$ & 0.7666 \\
\hline Decreased appetite/Anorexia & $8(2.49)$ & $9(2.79)$ & 1.0000 \\
\hline Fatigue & $7(2.18)$ & $9(2.79)$ & 0.8012 \\
\hline Dizziness & $7(2.18)$ & $9(2.79)$ & 0.8012 \\
\hline
\end{tabular}

AEs, adverse events.

intravenous fosaprepitant (Jiangsu Hansoh Pharmaceutical Group Co., Ltd.) for preventing CINV in Chinese patients treating with cisplatin-based chemotherapy.

On account of the different drug-delivery ways of aprepitant and fosaprepitant, we used simulation agents of both aprepitant and fosaprepitant to ensure purely double blinding. The rigorous designation of each treatment group receiving the same two modes of administration concurrently $(7,9)$, was of favorable significance to minimize the potential of study bias and error. It is worth noting that the different dexamethasone therapies were applied in the regimen of fosaprepitant group and aprepitant group especially on days 3 through 4, similar to the EASE study (9). Doses of dexamethasone administered on days 3 through 4 in addition to aprepitant were reduced according to the study drug schedule (Table 1), since previous reported data showed that aprepitant coadministration increases dexamethasone systemic exposure (16). As for efficacy, in the FAS, CR in the OP was $71.96 \%$ (95\% CI, $67.90 \%$ to $76.87 \%$ ) in fosaprepitant group in contrast to $69.35 \%$ (95\% CI, $64.32 \%$ to $74.38 \%)$ in aprepitant group ( $\mathrm{P}=0.4894$; Figure $2 A)$. A between-group difference of 2.61 percentage points was finally achieved (95\% CI, -4.42 to 9.64; Figure 3), where lower limit of $95 \%$ CI cis $-4.42 \%>-10 \%$ (noninferior limit), indicating that the fosaprepitant group is not inferior to the aprepitant group. Secondary endpoint of CR of fosaprepitant in the AP and DP was $90.97 \%(95 \%$ CI, $87.83 \%$ to $94.11 \%$ ) and $75.08 \%$ (95\% CI, $70.35 \%$ to
$79.81 \%$ ), which was also comparable to that of aprepitant with a $86.69 \%$ (95\% CI, $82.99 \%$ to $90.39 \%$ ) in the AP and $73.07 \%$ (95\% CI, $68.23 \%$ to $77.91 \%$ ) in the DP. Significantly, the PPS analysis was also consistent with the FAS, further supporting the solid evidence of these results. Our this current study of fosaprepitant in Chinese patients was well in accordance with the previous findings from Grunberg in achieving CR rates of $71.9 \%, 89.0 \%$ and $74.3 \%$ during the OP, AP and DP, respectively (9), identifying that a single-dose of $150 \mathrm{mg}$ with fosaprepitantbase (Jiangsu Hansoh Pharmaceutical Group Co., Ltd.) triple antiemetic regimen was noninferior to the standard aprepitant therapy. Accordingly, these results were also well consistent with the data from Asian population from Japan with CR rates of $64.2 \%, 93.6 \%$ and $64.7 \%$ in OP, AP and DP (7). However, another study investigating fosaprepitant-base (Chia Tai Tianqing Pharmaceutical Group Co., Ltd) triple antiemetic regimen in Chinese patients shown that a higher CR rates during the OP $(89.33 \%)$, as well as the AP (95.73\%) and DP (91.16\%) was achieved (23). The differences among these studies suggested that more data of fosaprepitant from Chinese patients were required to better evaluate and verify the efficacy and safety of various generic fosaprepitant in terms of CINV. As for safety profile, fosaprepitant (Jiangsu Hansoh Pharmaceutical Group Co., Ltd.) shown comparable tolerability to the aprepitant, the rates of treatment-related AEs and all the commonly reported AEs were similar between these two groups with no unexpected 
Table 4 Unit Cost and Sources

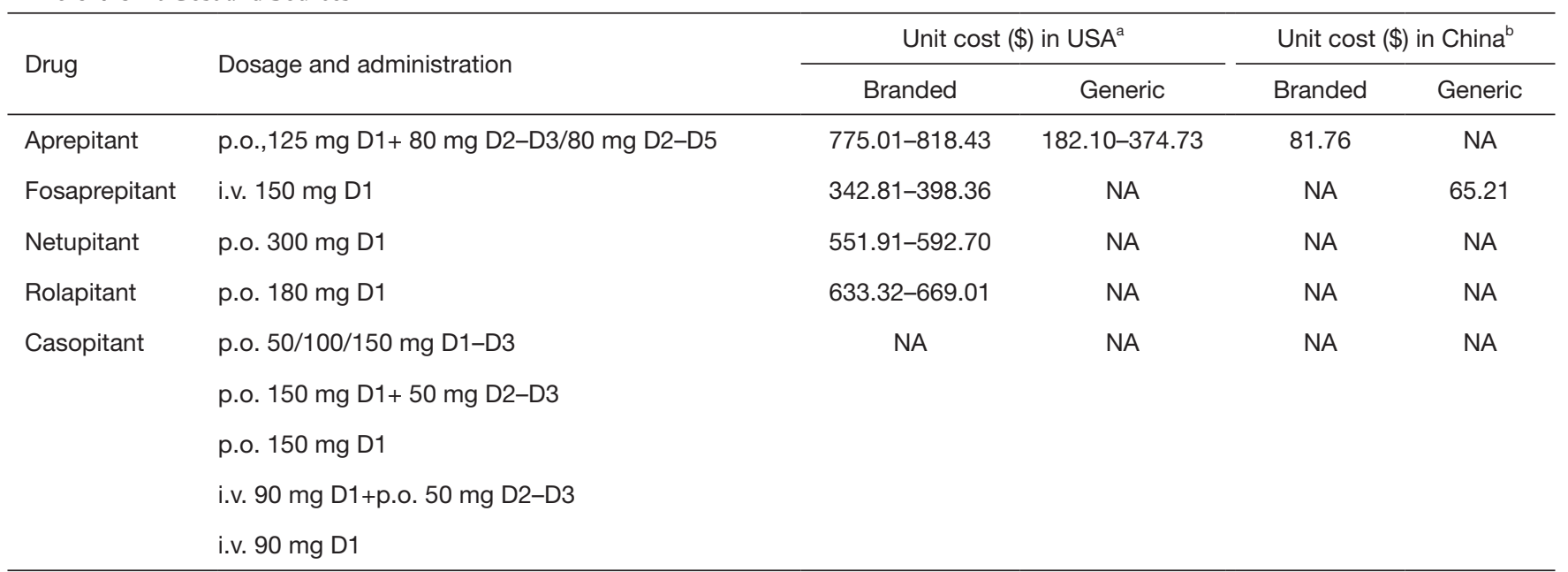

NA, not available. ${ }^{\text {aD }}$ rug price available from online RxUSA Pharmacy (http://rxusa.com), Drugs.com (https://www.drugs.com/price-guide/) and GoodRx (https://www.goodrx.com). ${ }^{b}$ Drug price available from online YAOZH (https://db.yaozh.com). 1 US dollar =7.0325 CNY (according to the latest exchange rate retrieved at 2019-12-01 23:15).

AEs were observed. No significant differences about the infusion site adverse events between these two treatment arms in this study $(\mathrm{P}>0.05)$ (Table S2).

Nowadays, NK-1 RA-based triple regimen are recommended as the standard therapy for patients receiving HEC and some MEC regimens. (NCCN, ESMO guidelines). However, although the NK-1 RA 1-based triple regimen bring improvement to the control of CINV, the price of NK-1 RA is a factor that must be taken into consideration in clinical practice. Prices of fosaprepitant and other NK-1 RA in the United States, Europe ranged from $\$ 182$ to $\$ 818$ (Table 4). The incremental cost of combining antiemetic prophylaxis needs further consideration, since it is relatively expensive for the general population. Many studies have shown that the high cost and insufficient medical insurance support are known barriers to comprehensive adoption of cancer therapy in clinical practice. The availability of high-quality generic drugs would benefit more patients as well as the sustainability of health-care systems by providing more treatment options with lower associated costs. Therefore, research and development into generic drugs is an urgent must for reducing drug prices and improving accessibility. To date, fosaprepitant was still not commercially available in China, leaving aprepitant as the only option for the current standard triple antiemetic regimen. Though the price of aprepitant has been modified at a reduced price about $¥ 575$, the lacking of intravenous formulated drug cause inconvenience to patients who were unable to uptake the orally formulated aprepitant. Therefore, high-quality research and development into generic fosaprepitant will be constructive to reducing the overall medical and economic burden of patients, especially in developing countries, and providing more feasible approach for certain population to obtain standard NK-1 RA 1-based triple regimen. From another angle, generic fosaprepitant can also lower the threshold of clinical research and make the upcoming antiemetic clinical trial more feasible and workable in the future.

This current study demonstrated a comparable efficacy and acceptable safety profile of generic fosaprepitant in contrast to the branded aprepitant in patients treated with HEC. Based on the results of this trial, the National Medical Products Administration (NMPA) has accepted the listing application of fosaprepitant (Jiangsu Hansoh Pharmaceutical Group Co., Ltd.) and is expected to be commercially available in China by the end of this year, with the aim to provide more alternatives for Chinese patients in the management of the distressing CINV.

\section{Conclusions}

Single-dose intravenous fosaprepitant $(150 \mathrm{mg})$ in combination with palonosetron and dexamethasone was well tolerated and demonstrated noninferior control of CINV to aprepitant-based triple regimen in Chinese patients treating with cisplatin-base chemotherapy. 


\section{Acknowledgments}

We thank the patients, their families and all the institutions and investigators who are participating in this study. We also thank the following investigators Guangyu An (Beijing Chao-Yang Hospital, Capital Medical University), Bangwei Cao (Beijing Friendship Hospital, Capital Medical University), Zhiyong Ma (Henan Cancer Hospital), Xiaodong Xie (General Hospital of the Northern Theater Command), Jingbo Kang (The Sixth Medical Center of PLA General Hospital), Yi Geng (Baoji Central Hospital), Jianhua Shi(Linyi Cancer Hospital) for participating in this study. This study was supported by Jiangsu Hansoh Pharmaceutical Group Co., Ltd.

Funding: Li Zhang has received research support from the Strategic Priority Research Program of the Chinese Academy of Sciences (No. XDA12020101 to J.D.). Yan Huang has received research support from Science and Technology Program of Guangzhou (201704020072). Yunpeng Yang was supported by Outstanding Young Talents Program of Sun Yat-sen University Cancer Center (16zxyc03) and Central Basic Scientific Research Fund for Colleges-Young Teacher Training Program of Sun Yat-sen University (17ykpy85).

\section{Footnote}

Conflicts of Interest: The authors have no conflicts of interest to declare.

Ethical Statement: The authors are accountable for all aspects of the work in ensuring that questions related to the accuracy or integrity of any part of the work are appropriately investigated and resolved. This trial has been approved by the institutional review boards (ID: 2014-xy060) and the independent ethics committees of Sun Yatsen University Cancer Center (Ethic approval ID: A2014058). And other participating centers have also obtained the approval from their institutional review boards/independent ethics committees before enrollment. All patients will be required to sign the informed consent form before enrollment. This study was also registered with www.china drugtrials.org.cn (ID: CTR20150598). Trial registration: CTR20150598 registered at www.chinadrugtrials.org.cn.

Open Access Statement: This is an Open Access article distributed in accordance with the Creative Commons Attribution-NonCommercial-NoDerivs 4.0 International
License (CC BY-NC-ND 4.0), which permits the noncommercial replication and distribution of the article with the strict proviso that no changes or edits are made and the original work is properly cited (including links to both the formal publication through the relevant DOI and the license). See: https://creativecommons.org/licenses/by-nc-nd/4.0/.

\section{References}

1. Oo TH, Hesketh PJ. Drug insight: New antiemetics in the management of chemotherapy-induced nausea and vomiting. Nat Clin Pract Oncol 2005;2:196-201.

2. Sommariva S, Pongiglione B, Tarricone R. Impact of chemotherapy-induced nausea and vomiting on healthrelated quality of life and resource utilization: A systematic review. Crit Rev Oncol Hematol 2016;99:13-36.

3. Hassan BA, Yusoff ZB. Negative impact of chemotherapy on breast cancer patients QOL - utility of antiemetic treatment guidelines and the role of race. Asian Pac J Cancer Prev 2010;11:1523-7.

4. Olver I, Ruhlmann CH, Jahn F, et al. 2016 Updated MASCC/ESMO Consensus Recommendations: Controlling nausea and vomiting with chemotherapy of low or minimal emetic potential. Support Care Cancer 2017;25:297-301.

5. Berger MJ, Ettinger DS, Aston J, et al. NCCN Guidelines Insights: Antiemesis, Version 2.2017. J Natl Compr Canc Netw 2017;15:883-93.

6. Lasseter KC, Gambale J, Jin B, et al. Tolerability of fosaprepitant and bioequivalency to aprepitant in healthy subjects. J Clin Pharmacol 2007;47:834-40.

7. Saito H, Yoshizawa H, Yoshimori K, et al. Efficacy and safety of single-dose fosaprepitant in the prevention of chemotherapy-induced nausea and vomiting in patients receiving high-dose cisplatin: a multicentre, randomised, double-blind, placebo-controlled phase 3 trial. Ann Oncol 2013;24:1067-73.

8. Weinstein C, Jordan K, Green SA, et al. Single-dose fosaprepitant for the prevention of chemotherapyinduced nausea and vomiting associated with moderately emetogenic chemotherapy: results of a randomized, double-blind phase III trial. Ann Oncol 2016;27:172-8.

9. Grunberg S, Chua D, Maru A, et al. Single-dose fosaprepitant for the prevention of chemotherapy-induced nausea and vomiting associated with cisplatin therapy: randomized, double-blind study protocol--EASE. J Clin Oncol 2011;29:1495-501.

10. Ando Y, Hayashi T, Ito K, et al. Comparison between 5-day 
aprepitant and single-dose fosaprepitant meglumine for preventing nausea and vomiting induced by cisplatin-based chemotherapy. Support Care Cancer 2016;24:871-8.

11. Maru A, Gangadharan VP, Desai CJ, et al. A phase 3 , randomized, double-blind study of single-dose fosaprepitant for prevention of cisplatin-induced nausea and vomiting: results of an Indian population subanalysis. Indian J Cancer 2013;50:285-91.

12. Alvarnas J, Majkowski GR, Levine AM. Moving Toward Economically Sustainable Value-Based Cancer Care in the Academic Setting. JAMA Oncol 2015;1:1221-2.

13. Djulbegovic B. Value-Based Cancer Care and the Excessive Cost of Drugs. JAMA Oncol 2015;1:1301-2.

14. Young RC. Value-Based Cancer Care. N Engl J Med 2015;373:2593-5.

15. Hu Z, Cheng Y, Zhang H, et al. Aprepitant triple therapy for the prevention of chemotherapy-induced nausea and vomiting following high-dose cisplatin in Chinese patients: a randomized, double-blind, placebo-controlled phase III trial. Support Care Cancer 2014;22:979-87.

16. McCrea JB, Majumdar AK, Goldberg MR, et al. Effects of the neurokinin1 receptor antagonist aprepitant on the pharmacokinetics of dexamethasone and methylprednisolone. Clin Pharmacol Ther 2003;74:17-24.

17. Grunberg SM, Boutin N, Ireland A, et al. Impact of nausea/vomiting on quality of life as a visual analogue scale-derived utility score. Support Care Cancer 1996;4:435-9.

Cite this article as: Zhang Z, Yang Y, Lu P, Li X, Chang J, Zheng R, Zhou L, Chen S, Chen X, Ren B, Gu W, Jiang X, Peng J, Huang M, Feng G, Shen P, Zhang Q, Zhang B, Huang Y, He J, Chen Y, Cao J, Wang H, Li W, Wan H, Nan K, Liao Z, Zhang C, Lin Z, Zhong D, Xu Q, Liu H, Sun T, Deng Y, Zhang L. Fosaprepitant versus aprepitant in the prevention of chemotherapy-induced nausea and vomiting in patients receiving cisplatin-based chemotherapy: a multicenter, randomized, doubleblind, double-simulated, positive-controlled phase III trial. Ann Transl Med 2020;8(5):234. doi: 10.21037/atm.2019.12.158
18. Decker GM, DeMeyer ES, Kisko DL. Measuring the maintenance of daily life activities using the functional living index-emesis (FLIE) in patients receiving moderately emetogenic chemotherapy. J Support Oncol 2006;4:35-41, 52.

19. Feyer P, Jordan K. Update and new trends in antiemetic therapy: the continuing need for novel therapies. Ann Oncol 2011;22:30-8.

20. Aapro M, Molassiotis A, Dicato M, et al. The effect of guideline-consistent antiemetic therapy on chemotherapyinduced nausea and vomiting (CINV): the Pan European Emesis Registry (PEER). Ann Oncol 2012;23:1986.

21. Warr DG, Grunberg SM, Gralla RJ, et al. The oral NK antagonist aprepitant for the prevention of acute and delayed chemotherapy-induced nausea and vomiting: Pooled data from 2 randomised, double-blind, placebo controlled trials. Eur J Cancer 2005;41:1278-85.

22. Warr DG, Hesketh PJ, Gralla RJ, et al. Efficacy and tolerability of aprepitant for the prevention of chemotherapy-induced nausea and vomiting in patients with breast cancer after moderately emetogenic chemotherapy. J Clin Oncol 2005;23:2822.

23. Yang LQ, Sun XC, Qin SK, et al. Efficacy and safety of fosaprepitant in the prevention of nausea and vomiting following highly emetogenic chemotherapy in Chinese people: A randomized, double-blind, phase III study. Eur J Cancer Care (Engl) 2017;26. doi: 10.1111/ecc.12668. 


\section{Supplementary}

\section{Efficacy}

Kaplan-Meier curves of time to first vomiting failure were similar between the two groups ( $\mathrm{P}=0.8110$, Figure S1). The proportions of receiving rescue therapy during the $\mathrm{OP}$ were $5.61 \%$ vs. $7.74 \%(\mathrm{P}=0.3438)$ in the fosaprepitant and aprepitant groups, respectively. Furthermore, according to FLIE, scores of no impact of CINV on daily life had no significant differences in these two therapeutic groups before and after treatment $(\mathrm{P}>0.05)$. The change of FLIE score before and after treatment in the fosaprepitant group was $-9.73 \pm 21.55$, with statistical significance $(\mathrm{P}<0.0001)$; the change of FLIE score before and after treatment in the aprepitant group was $-9.93 \pm 18.76$, with statistical significance $(\mathrm{P}<0.0001)$.

\section{Tolerability}

There were 2 cases of injection pain, 1 case of phlebitis, and 1 case of vessel puncture site pain in patients receiving fosaprepitant regimen. While in patients receiving aprepitant regimen, 1 case of phlebitis and 1 case of injection site reaction were reported. There were no significant differences about the infusion site adverse events between the two treatment arms in this study $(\mathrm{P}>0.05)$ (Table S2). With respect to vital signs and laboratory tests, there were no significant differences in the effects between fosaprepitant and aprepitant on both the vital signs (respiration, body temperature, weight and blood pressure) $(\mathrm{P}>0.05)$ and the laboratory tests of the patients $(\mathrm{P}>0.05)$.

Table S1 Subgroup analysis of univariate logistic regression of CR rate in the overall phase $(0-120 \mathrm{~h})$ in FAS

\begin{tabular}{|c|c|c|c|c|c|c|c|}
\hline Subgroup & B & SE & $\chi^{2}$ & OR & \multicolumn{2}{|c|}{ OR $95 \% \mathrm{Cl}$} & $P$ value \\
\hline \multicolumn{8}{|c|}{ History of chemotherapy } \\
\hline Never & -0.1062 & 0.209 & 0.2581 & 0.899 & 0.597 & 1.354 & 0.6114 \\
\hline Ever & -0.1901 & 0.3119 & 0.3716 & 0.827 & 0.449 & 1.524 & 0.5421 \\
\hline Male & -0.1114 & 0.2361 & 0.2225 & 0.895 & 0.563 & 1.421 & 0.6371 \\
\hline Female & -0.1431 & 0.2585 & 0.3067 & 0.867 & 0.522 & 1.438 & 0.5797 \\
\hline \multicolumn{8}{|l|}{ Age } \\
\hline$<55$ & -0.224 & 0.2602 & 0.7416 & 0.799 & 0.48 & 1.331 & 0.3892 \\
\hline 0 & -0.4166 & 0.3062 & 1.8507 & 0.659 & 0.362 & 1.202 & 0.1737 \\
\hline $1-2$ & 0.0113 & 0.2111 & 0.0029 & 1.011 & 0.669 & 1.530 & 0.9572 \\
\hline
\end{tabular}

CR, complete response; FAS, full analysis set; OR, odds ratio; LL, lower limit; UL, upper limit; ECOG, Eastern Cooperative Oncology Group.

Table S2 Patients with infusion site adverse events

\begin{tabular}{lccc}
\hline Infusion site adverse event, $\mathrm{n}(\%)$ & Fosaprepitant regimen $(\mathrm{n}=321)$ & Aprepitant regimen $(\mathrm{n}=323)$ & $\mathrm{P}$ value \\
\hline Injection site pain & $2(0.62)$ & $1(0.00)$ & 0.2481 \\
Phlebitis & $1(0.31)$ & $0(0.00)$ & 1.0000 \\
Vessel puncture site pain & $1(0.31)$ & $1(0.31)$ & 0.4984 \\
Infusion site reaction & $0(0.00)$ & 1.0000 \\
\hline
\end{tabular}




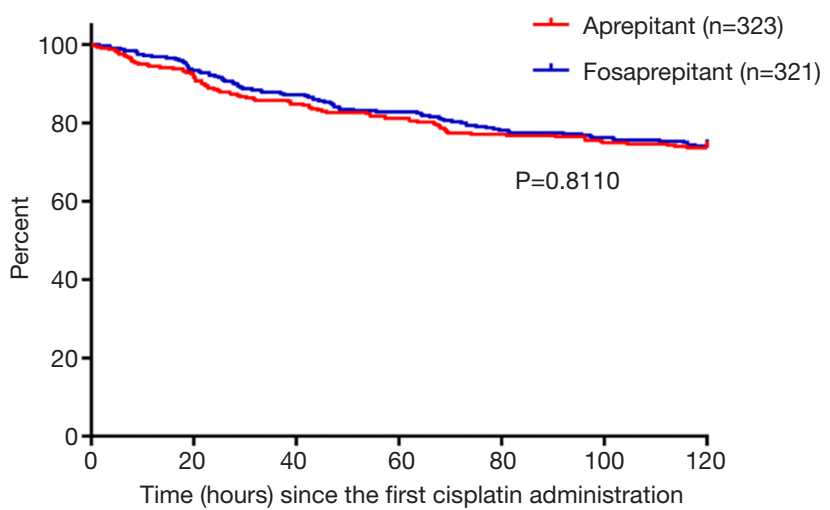

Figure S1 Kaplan-Meier curves of time to first vomiting failure. 